(anterior cingulotomy) or entry to the DBS trial. OCD medication was kept constant throughout the trial. We tested the effects of DBS by comparing baseline, VC/VS, STN, both sites and CBT stages of trial on the following three assessments of function: GAF, SDS and CAOIC. An impairment focussed interview was done to quantify changes in function specific to OCD. Friedman's test was used to test for DBS effects during the double-blind crossover phases comparing baseline, amSTN and VC/VS. Post-hoc pair-wise Conover tests for significant effects were used with FDR corrections. All significant results are reported at $\mathrm{P}<0.05$.

Result. DBS had a significant effect across all phases for all above mentioned clinical measures. For all three measures of disability, there were significant improvements after both amSTN and VC/ VS DBS. For all three measures of disability the effect of VC/VS DBS was significantly better that amSTN DBS. For all three measures of disability there was a non-significant trend $(p=0.058)$ for stimulation at both sites to have a better effect than stimulation of one site alone. For all three measures of disability, there were no significant difference between DBS alone and DBS and CBT.

Conclusion. This study is the first to have directly compared differential effects of STN versus VC/VS DBS stimulation in OCD patients whilst testing clinical, cognitive and disability outcomes. The results of this study indicate that although both sites are equally effective in reducing OCD, stimulating VC/VS leads to a significantly greater improvement on disability scores in severe OCD.

\section{Review: Psychological \& Behavioural Treatments of Nonepileptic Seizures in Children and Adolescents}

Hemma Velani* and Julia Gledhill

Central and North West London NHS Foundation Trust ${ }^{*}$ Corresponding author.

doi: 10.1192/bjo.2021.791

Aims. To systematically review Psychological and Behavioural treatments on NES in children and adolescents by reviewing the current literature.

Background. Non-epileptic seizures (NES) are associated with a high level of functional impairment for young people and their families. However, there are no UK guidelines for the management of NES in children and adolescents or adults. Though information from the limited studies in adults may be useful, the findings may not be generalizable to children and adolescents. To date, we are unaware of any published systematic review on this topic in children and adolescents.

Method. A systematic search of relevant electronic databases was conducted. Any study investigating the effectiveness of psychological and behavioural treatments on NES, in Children and Adolescents was included.

Result. Fifteen studies were identified, but only six studies had the primary aim of evaluating an intervention, and only one used a control group. The rest were observational studies that examined retrospective case notes.

CBT and psychoeducation were identified as the most common interventions. Eleven out of the fifteen studies used multiple treatments, four looked at one treatment only, three of these CBT and one was a natural history study.

Where individual therapy was provided, a common focus was management of anxiety, usually delivered in a flexible way, adapted to individual needs. Despite being identified as important in the literature, only one study demonstrated care that involved collaboration between physical and mental health teams.
Conclusion. It's difficult to conclude from this review that one treatment approach is superior to another. The findings of this review offer some insight into current practise and may help to inform future research in this area. CBT and psychoeducation with a focus on anxiety are frequently included in interventions for NES in young people, and further evaluation of these treatment modalities could be a helpful next step.

\section{The experience of patient dropout from eating disorders treatment: a systematic review and qualitative synthesis}

Cecilia Vinchenzo ${ }^{1 *}$, Catherine $\mathrm{McCombie}^{2}$ and Vanessa Lawrence ${ }^{2}$

${ }^{1}$ IoPNN King's College London, Lancaster University and ${ }^{2}$ IoPPN King's College London

${ }^{\star}$ Corresponding author.

doi: $10.1192 /$ bjo.2021.792

Aims. Eating disorders are serious and debilitating mental health conditions associated with high relapse and mortality rates and significant psychiatric comorbidities. Research suggests approximately $50 \%$ of patients with an eating disorder dropout prematurely from treatment services, fostering poorer health outcomes and impacting significantly on patients, their families, health services and research quality. The aim of this review is to synthesise the current qualitative literature available on the patient experience of dropout from eating disorder services and understand the reasons motivating early treatment termination.

Method. A systematic search was carried out and articles selected from MEDLINE, PsycINFO, EMBASE and CINAHL. Studies were included if they explored eating disorder treatment dropout using qualitative data collection or analysis methods. Study quality was critically appraised using the Critical Appraisal Skills Programme qualitative research evaluation tool. Thematic synthesis was used to interpret and synthesise themes from the primary studies.

Result. Ten studies met the inclusion criteria for the systematic review. Five studies were scored as high quality and five as medium quality. 13 descriptive sub-themes encompassing the dropout experience were identified under four overarching analytical themes: inner conflict, connection and communication with others, experience of the treatment service, and factors related to progress in treatment.

Conclusion. The decision to drop out from eating disorder treatment is a complex, multi-faceted issue, involving an interplay between individual, social and service-level factors. This review highlights the need for further high quality qualitative investigation into dropout experiences, with an effort to increase representation across ethnic groups and gender identities. This review also identifies the need for a reconsideration of current clinical practice and services provision with an emphasis on the use of patient perspectives to guide decision making in eating disorder services delivery and research. Moreover, the findings emphasise the need for standardised dropout definitions, fostering a unified literature base.

Patient experiences of the pandemic; exploring the effect of COVID-19 on patients detained under the Mental Health Act

\section{Emily Watson* and Samuel Rowles}

St Andrews Healthcare

${ }^{*}$ Corresponding author.

doi: 10.1192/bjo.2021.793 